\title{
Putting pregnancy track in hands of women to try to reduce Maternal-fetal morbidity and mortality
}

\author{
Antonio Godoy, Diego Mendoza, Jesús Campos, Lizbeth Escobedo \\ Engineering School \\ CETYS Universidad \\ Tijuana, Baja California, México \\ antonio.godoy@cetys.edu.mx,diego.mendoza@cetys.edu.mx,jesus.campos@cetys.edu.mx \\ lizbeth.escobedo@cetys.mx
}

\begin{abstract}
Historically woman's' health during pregnancy is in hands of doctors. Through monthly clinical visits women are informed about their health. But not all woman have the opportunity to on-site clinical visits or/and woman need constantly support in this hard and unknown journey. This lack of support is worldwide rising the maternal-fetal morbidity and mortality. This paper shows a study towards the design of PREGLOG, a mobile application purely thought for pregnant women to help them overcome any situation which could complicate their pregnancy process, including anomaly logs and useful information about the symptoms they could present during their current pregnancy month. We interviewed specialists in charge of maternal control of women, including three gynecologists and a specialist in Family Medicine, and 10 pregnancies in order to understand pregnancy care and pregnancy doubts, fears, and needs. As a result we obtained valuable insights that will help us to design a prototype which fulfills the main needs for both the patients and the specialists who treat them.
\end{abstract}

\section{CCS CONCEPTS}

- Human-centered computing $\rightarrow$ Human computer interaction $(\mathrm{HCI}) \rightarrow$ Empirical studies in $\mathrm{HCI}$

\section{KEYWORDS}

Pregnancy health, mobile application, pervasive health

\section{INTRODUCTION}

During pregnancy complications may occur but most of them can be prevented or treated. The part of the treatment always remains in the responsibility of medical attention. According to WHO ("WHO Recommendations on Antenatal Care for a Positive Pregnancy Experience" n.d.) a pregnant need an on-site clinical visit by month, and by week in the last month. The women who perform with the monthly clinical visit are given the word that is touched in the treatment, but this is not the case for all pregnancies.
Pregnancies face many fears and doubts daily during pregnancy, this scenario multiplies for woman in rural zones where they don't have access to at least monthly on-site clinical visits.

There are some apps for pregnancy monitoring that allow woman to visualize the baby's development, to use a calendar for clinical visits, read articles about pregnancy, add notes, etc. That is why a mobile application can be of great support for the personal monitoring of a pregnancy, which can prevent or announce an event or anomaly that the patient presents. The main problem is that year after year a large number of maternal and fetal deaths occur (Filippi et al. 2016; Hirshberg and Srinivas 2017), which is caused by the little or no follow-up given to the process of a pregnant woman, and by the lack of information from the patients. It is necessary that both parties (e.g., pregnant women and specialists) work together and are in constant communication with the purpose of carrying the most detailed precise monitoring of pregnancy. Patients should be informed about the good and the bad things that could happen to woman's health that can derive a problem to either mom or baby during pregnancy.

Over the years there have been mobile applications whose purpose is to support pregnant women to record information related to their pregnancy and show what the progress of their baby's formation should be. However, these applications have not covered the problem we are attacking, in addition, they have not met the users' needs (e.g., pregnant women) and they have been forgotten.

We present here the results of a formative study where we collected data from gynecologists and pregnant women. The aim of these investigations was to give to pregnancies support to monitor their health and thus try to reduce maternal-fetal morbidity and mortality. We envision the design of a high-fidelity prototype in order to develop a mobile application, which would be the day-today support in the life of a pregnant woman.

\section{RELATED WORK}

During the compilation of information, different contributions were found which were related to the topic, but only one called "AESOP: Progress of Pregnancy"1, was the most similar to our

\footnotetext{
${ }^{1}$ http://www.aesopapps.com/pregnancyprogress/
} 
research. We inquired about the functionality of this application where the following are described:

- Make your pregnancy easier and more pleasant.

- Follow your progress and save your memories.

- Share the memorable moments of your pregnancy with friends and family in a simple way.

- Record your medical appointments, questions and symptoms.

- Check calendars and tables to follow the progress of your pregnancy.

- Perfect to make your pregnancy easier and more pleasant as you count the days to your due date.

The application seems to have a good goal, but according to Apple's App Store users who opted to buy this application for 2.30 Euros and left their review made the application average 1.2 stars out of 5 possible. In the reviews you can see what users commented on what the application it is not well developed in its entirety, due to the fact that its features mentioned in its descriptions do not work correctly. What generates that the application is not optimized to give the suitable follow-up of the pregnancy.

This is taken as a lesson for our research, the application tries to cover too many fields and it is not user-oriented, which may have resulted in an error for them. So you can see these applications deal with the beautiful part about pregnancy, but we are not given information about anomalies, alert cases, risks and other alarming factors that should not be overlooked during pregnancy.

\section{METHODS}

\subsection{Participants Description}

Three different gynecologists and a specialist in Family medicine were interviewed, each one had a different degree of experience, and the support of the participants was of vital importance to enter in the context of the great topic that is being treated. During the interviews with them, we addressed different topics, such as the characteristics that a patient presents, the pregnancy process, the diagnosis of a pregnancy, treatment, monitoring and self-evaluation, etc.

On the other hand, 10 pregnant women were interviewed, as well with different ages, and with/without previous experience on a pregnancy process. We wanted to know and understand how much women know about the factors that must be taken into account during their pregnancy. We also discussed what they would like to get from a mobile application, which was very important for the design stage.

\subsection{Data Collection}

We decided to divide the data collection process into 2 stages, on which we applied both qualitative and quantitative data collection techniques, such as interviews, focus group in order to retrieve all the necessary information to continue with the project.

(1) Investigation: In this stage, we investigated everything we could about the topic during two weeks. This includes terminology, the pregnancy process, symptoms, anomalies, etc. We dived deep into the topic in order to be comfortable with the information we were manipulating before meeting with the specialists and the pregnant women.

(2) On-field data collection: After two weeks of intense investigation, and 3 iterations with the support of an HCI expert, we created one interview protocol for specialists, consisting of 23 questions. After creating the interview protocol we got 3 interviews. The first 2 interviews were conducted in the gynecologists' personal office. The third interview was performed in the reproductive and fertility clinic, with gynecologist expert in fertility.

Each of the interviews was recorded with audio with the consent of the participants and conducted in an average time of 30 minutes. The protocol designed for the specialists was very specific about the topic, we focused on collecting data about the symptoms, anomalies, the process of monitoring, what they do on every session, how do they know if the baby and the woman are doing good on their process, etc.

Contrarily, the protocol designed for the pregnant women was much less specific, as we only wanted to know how do they feel during their pregnancy process, what kind of information they manage, and where do they get it from.

With the information already collected from the gynecologists, and after analyzing it, it was possible to create a visions of a lowfidelity prototype following the recommendations of both specialists and pregnant women. The purpose of these visions was to show the users what kind of information they would be able to see on the screens (e.g., such as symptoms according to your pregnancy month) and the functions they would be able to perform (e.g., such as register anomalies and getting feedback with recommendations about how to proceed according to the anomaly registered).

Once this was done, we could design a first sketch, whose purpose was to capture the main functionalities of the application. This was possible thanks to the information gathered with the gynecologists. However, it was necessary to have the opinion of those who will use the application. The interview for pregnant women took over 2 weeks, and it consisted on 18 questions, each of them was carried out in an average of 8-12 minutes. In this case no audio was recorded in order to avoid any inconvenience with the interviewees.

\subsection{Data Analysis}

According to the information collected from the gynecologists we compared each question-answer in order to discover insights on the data. On the other hand, with the pregnant woman we used a different method to analyze the results. As we interviewed 10 woman, we created a temporal spreadsheet to know the more common answers from them and discover useful insights for the application. All of the insights we found are listed on the following section (Results).

\section{RESULTS}

\subsection{Gynecologists Insights}


After analyzing the surveys and talks given by the gynecologists, we found the following insights:

(1) The average age range of pregnant women who are monitoring their pregnancy with a specialist is between 20 and 45 years old.

(2) Any of the following factors must be present in a pregnancy in order to be considered as a high-risk pregnancy:

- Age $<20$ years or $>35$ years.

- Less than 2 years of recovery between two pregnancies.

- History of 4 pregnancies or more.

- Antecedents of abortions, hemorrhages, fetal deaths, prematurity, arterial hypertension, preeclampsia, cesarean or diabetes in previous pregnancies.

- Congenital malformations.

- Cardiomyopathy.

- Nephropathy.

(3) The level of urgency of a pregnant patient is measured according to their symptoms. Within this symptomatology are key points which are critical and the pregnancy is considered an immediate emergency when any of these are present:

- Abdominal pain.

- Bleeding.

- Vaginal flow.

- Lack of movement of the baby.

- Headache.

- Ringing of the ears.

- Discomfort in light.

- Swollen feet.

(4) The most common symptoms for which a patient goes to the emergency room or to an emergency appointment are abdominal pain, bleeding and fever.

(5) The most common diseases that are generated during a pregnancy are gestational diabetes, preeclampsia, urinary tract infection.

(6) Regarding the monitoring of the patient, one of the aspects that takes great care is the constant review of the weight of the patient as well as the baby. A considerable increase in any of these may indicate the onset of gestational diabetes (this normally occurs in the 2 nd and 3rd semester of pregnancy). At the same time blood and urine studies are added, which are carried out at the beginning of each trimester of pregnancy (in a high risk pregnancy this changes and more serial studies are carried out to detect any anomaly in time).

(7) The best way to help reduce maternal-fetal morbidity and mortality is to inform the patient about the negative situations that may occur during pregnancy, what they mean and how to react in order to avoid negligence from the patient.

(8) Regarding what was discussed with the gynecologists about the application, as mentioned on the Data Analysis section, some suggestions were given:

- Questionnaire available for any woman who wants to get pregnant in order to discover if their current conditions are optimal to get pregnant.
- Avoid notifications system for gynecologist, they argued that it will saturate their smart phones, which is already saturated from conversations with their patients.

- Add a sort of 'diary' where the patient could add any anomaly, therefore when they go with their gynecologist for the scheduled session, they do not have to remember every anomaly they presented, instead they would just check that diary.

- Classification based on the symptomatology described as critical above.

\subsection{Pregnant Women Insights}

After collecting the data with the pregnant women, the following results were obtained:

(1) As the specialists commented, the age range is between 20 and 35 years, with a slight increase for risk in women older than 35 years old

(2) The women interviewed were mostly on their first or second pregnancy process.

(3) Only $50 \%$ of our interviewees attend with a gynecologist, the other $50 \%$ is divided in attending a general practitioner or just relying on their own experience or knowledge.

(4) Patients who attend with a gynecologist or a doctor in general, usually go once a month.

(5) In their totality, all the women commented that it is important to attend with a gynecologist.

(6) Women manage to detect the most common symptoms such as bleeding or high blood pressure, but mostly symptoms such as headache, discomfort in light, or swelling of the feet, are not considered as symptoms to be alarmed about for them. Therefore, it is confirmed that the lack of information can be the biggest enemy in an emergency situation.

(7) 7 out of the 10 interviewed people had a high risk pregnancy, and only 2 of them did not know. In this area, it can be interpreted that they are a little more informed about the topic, however, the problematic is found in the anomalies that occur during their pregnancy process.

(8) They consider that an application where they can monitor their own health with data in their hands would be very useful for monitoring their pregnancy.

(9) Mostly all of the pregnant women spent 2 to 3 hours using their smart phone.

(10) They regularly look for information about their pregnancy on the internet.

(11) They would like to have an application to use it only whenever they need information about their pregnancy. No push notifications.

(12) They would like to be notified by the application about the symptoms that occur during their current pregnancy month and how they should react to any of them.

(13) On average, women would pay from $\$ 1$ to $\$ 5$ US dollars for the application.

\section{CONCLUSIONS}

We have shown the results of this research, which was based on the interview with 3 gynecologists, a specialist in Family Medicine 
and 10 pregnant women. All of these sources provided useful information for the research and for the design of PREGLOG, a high-fidelity prototype of our proposal, which consists of developing an application that supports the reduction of maternalfetal morbidity and mortality. We found that disinformation is really the one that endangers the lives of mothers and their babies, most of the cases where a loss is obtained, whether maternal or fetal, could have been avoided if the disinformation would have been treated from the beginning.

In addition, the team believes that this project could bet the basis for further researches on this subject, due to the fact that it is a very broad field. We also found out that combining this problem with computer science was very useful because we were able to attack it implementing an innovative and not so traditional proposal, as we live in an era where the smart phone is part of the daily life of humans, we decided to face this problem with a mobile application.

To conclude with this paper, as a work in the future we have thought about how this application can be implemented and extended. We would like to create a link between the patients and the gynecologists within the application, adding functionalities such as detecting a high risk pregnancy from the beginning, monthly generated reports for both the patient and the gynecologist, among many others. We are also thinking about attacking other problems in different treatments, not only in a pregnancy, it can be the case of the treatment of some disease, or some specialized follow-up that is needed.

\section{ACKNOWLEDGMENTS}

The authors would like to thank participants of this study (both gynecologists and pregnant women) for their valuable comments and helpful suggestions.

\section{REFERENCES}

[1] Filippi, Véronique, Doris Chou, Carine Ronsmans, Wendy Graham, and Lale Say. 2016. Levels and Causes of Maternal Mortality and Morbidity. Reproductive, Maternal, Newborn, and Child Health: Disease Control Priorities, Third Edition (Volume 2). The International Bank for Reconstruction and Development / The World Bank. https://doi.org/10.1596/978-1-4648-0348-2_CH3

[2] Hirshberg, Adi, and Sindhu K. Srinivas. 2017. "Epidemiology of Maternal Morbidity and Mortality." Seminars in Perinatology 41 (6):332-37. https://doi.org/10.1053/j.semperi.2017.07.007.

[3] WHO Recommendations on Antenatal Care for a Positive Pregnancy Experience." n.d. Accessed January 15, 2019. http://apps.who.int/iris/bitstream/handle/10665/250796/9789241549912eng.pdf;jsessionid=FB3CB4EC01D5928BFDD38B1AD3C82E3F?sequence $=1$ 